\title{
Experimental Assessment of Harmful Interference Between Broadcasting and Distributed Cognitive Radio Networks in Real Scenarios
}

\author{
$1^{\text {st }}$ Rodney Martinez Alonso \\ Information Technology \\ Ghent University \\ Ghent, Belgium \\ rodney.martinezalonso@ugent.be
}

\author{
$2^{\text {nd }}$ David Plets \\ Information Technology \\ Ghent University \\ Ghent, Belgium \\ david.plets@ugent.be
}

\author{
$3^{\text {rd }}$ Arley Coto Guerra \\ Research and Development \\ LACETEL \\ Havana, Cuba \\ arley@lacetel.cu
}

\author{
$4^{\text {th }}$ Yosvany Hervis Santana \\ Information Technology \\ Ghent University \\ Ghent, Belgium \\ yosvany.hervissantana@ugent.be
}

\author{
$5^{\text {th }}$ Ernesto Fontes Pupo \\ Research and Development \\ LACETEL \\ Havana, Cuba \\ fontes@lacetel.cu \\ $6^{\text {th }}$ Alejandro Gonzalez Garcia \\ Research and Development \\ LACETEL \\ Havana, Cuba \\ alejandro@lacetel.cu
}

\author{
$7^{\text {th }}$ Rufino Cabrera Alvarez \\ Research and Development \\ LACETEL \\ Havana, Cuba \\ rufino@lacetel.cu
}

$8^{\text {th }}$ Glauco Guillen Nieto

Research and Development

LACETEL
Havana, Cuba
glauco@lacetel.cu

\author{
$9^{\text {th }}$ Luc Martens \\ Information Technology \\ Ghent University \\ Ghent, Belgium \\ luc1.martens@ugent.be
}

\author{
$10^{\text {th }}$ Wout Joseph \\ Information Technology \\ Ghent University \\ Ghent, Belgium \\ wout.joseph@ugent.be
}

\begin{abstract}
Spectrum scarcity has become a challenge for the growing demand of wireless services. Paradoxically, although most of the sub-1-GHz spectrum is assigned it is underutilized in the space and time domain. Several surveys demonstrate a spectrum usage efficiency lower than $20 \%$. Cognitive Radio technologies has been proposed as a long-term solution for tackling spectrum scarcity and inefficiency. However, a major concern has been the interference to and from the primary licensed service. In this paper we investigate the coexistence between broadcasting digital television and LTE-A distributed cognitive radio in real scenarios. Our research revealed that, for the worst case the LTE-A distributed cognitive radio causes a degradation of the broadcasting service QoE higher than $60 \%$. For the LTE-A network there is up to $5 \mathrm{~dB}$ C/I margin that does not worsen its QoS, being a potential resource for networking optimization and interference mitigation.
\end{abstract}

Index Terms-Cognitive Radio, Broadcasting, Coexistence, Quality of Experience, Quality of Service

\section{INTRODUCTION}

The growing demand for broadband wireless communications, new services and applications, and the static management of the spectrum altogether are limiting technology efficiency and affecting economic network performance indicators [1]. Paradoxically, although most of the sub- $1 \mathrm{GHz}$ spectrum is assigned, it is underutilized in the space and time

This paper has been supported in part by a grant from the Special Research Funds allocated by the Flemish Government to Ghent University, by LACETEL, the Cuban Ministry of Communications and Rhode \& Schwarz, Belgium. domain. Several surveys demonstrate a spectrum usage efficiency lower than 20\% [2]-[7]. This inefficient management of the spectrum is causing the need for higher frequency band allocation for new services and applications. As a direct consequence of this higher frequency, the cell coverage range is reduced, requiring more base stations to cover the service area. In this way, CAPEX and OPEX increase, reducing the affordability in suburban and rural areas with low population density.

Cognitive radio technologies enabling dynamic spectrum access, have become a more efficient alternative for spectrum management. Cognitive radio technologies allow sharing a certain frequency band by different services. The spectrum that is allocated by the regulatory domain to a primary service, can be used by secondary services under the condition that they do not interfere with the primary one at a certain location and given time [1]. Hence, the underutilized spectrum can be reused by other devices without needing a fixed spectrum band assignment by the regulatory domain [8]. This has led to more efficient usage of the spectrum.

The relevance of dynamic spectrum access based on cognitive radio technologies for enabling cost-effective wireless networks was acknowledged by the European Telecommunications Standards Institute (ETSI) and the Third Generation Partnership Project (3GPP) [9]. In the technical report TR 103067 [8] the ETSI analysed the feasibility of LTE Cognitive Radio Systems operating TVWS spectrum. Also, 
typical use cases for the operation of LTE re-configurable radio in TVWS and methods for avoiding harmful interference have been analysed and proposed by ETSI technical report TR 102907 [10]. Dynamic spectrum access in 4G networks is not exclusive for TVWS. For instance, the LongTerm Evolution - Unlicensed (LTE-U) standard allows the shared use of channels in the unlicensed ISM band with technologies like WiFi and Bluetooth, mainly for mitigating congestion issues in the $4 \mathrm{G}$ mobile networks and the lack of available spectrum [11]. A similar approach was announced by Ericsson for the dynamic spectrum assignment in the mobile network infrastructure, depending on the density of $4 \mathrm{G}$ and $5 \mathrm{G}$ users [12].

The spectrum-sensing-based techniques in the traditional cognitive radio networks could lead to unwanted harmful interference caused by the lack of network optimization or hidden nodes, i.e., when a cognitive radio device cannot accurately sense the broadcasting signal, but its emissions interfere with the broadcasting service [13]. This problem can be minimized by using a centralized access controller receiving information from all network devices [14].

In this paper, via a subjective measurement campaign, we quantify the QoE degradation at the broadcasting network coverage edge, when an LTE-A Customer Premise Equipment (CPE) or Base Station (BS) is operating in the same channel at the broadcasting transmitter protection contour. We assume that a cognitive radio application for the latest LTE-A release is deployed. Also, we assess the throughput degradation of the LTE-A network as a function of the broadcasting interference.

\section{METHOD}

A. Experimental assessment of the broadcasting service QoE degradation caused by LTE-A interference

The goal of our experiment is to quantify the percentage of video degradation caused by an LTE-A device operating at the protection contour of the broadcasting transmitter. These measurements are performed in the suburban region of Ghent, East Flanders, Belgium and Boyeros, Havana, Cuba. Fig. 1 shows the scenario model for emulating the critical interference conditions from the LTE-A network to the broadcasting service.

We define the coverage edge of the transmitter for the current broadcasting transmitters' configuration in Ghent and Boyeros, for $90 \%$ of locations covered $99 \%$ of the time. For DTMB [15] this corresponds to a signal level of $-84 \mathrm{dBm}$ [16] and $-83 \mathrm{dBm}$ for DVB-T2 [17]. The protection contour limit is defined for a median signal level of $-95 \mathrm{dBm}$. Here, we investigate the worst-case scenario, being an LTE-A device radiating the maximum allowable power level at the edge of the broadcasting transmitter protection contour (red diamond in Fig. 1). For Boyeros propagation conditions, the median interference level from the LTE-A device at the cell edge of the broadcasting transmitter is $-104 \mathrm{dBm}$ and $-106 \mathrm{dBm}$ for Ghent. The interference scenario at the coverage edge of the broadcasting transmitter were emulated in laboratory by adding in a closed loop a locally generated broadcasting

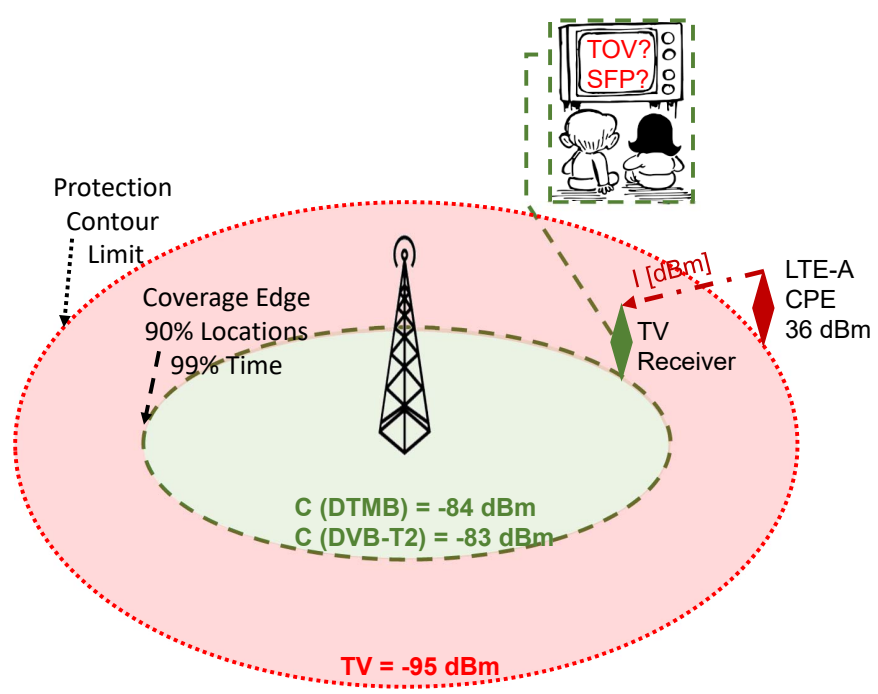

Fig. 1. Scenario Model: interference from LTE-A cognitive radio to a real Broadcasting network.

signal with the on-the-air LTE-A signal. The LTE-A signal was properly adjusted for the levels described for this experiment. The percentage of load of the BSs will correspond to the real traffic generated by the users at different periods of time.

We perform a subjective quality test at the cell edge of the broadcasting transmitter (Fig. 1). For the experimental assessment we consider two subjective indicators, i.e., the Threshold of Visibility (TOV) defined by the Report ITU-R BT.2035-2 [18] and the Subjective Failure Point (SFP) defined by the Recommendation ITU-R BT.1368-13 [19]. The total observation time of 40 minutes is divided in time stamps of 1 minute (TOV) or 20 seconds (SFP). For each time stamp we record when the TOV or SFP is exceeded. The test is repeated at different times of the day for accounting for different traffic loads in the LTE-A BS, i.e., a load of $10-20 \%, 30-40 \%$, 50$60 \%, 80-90 \%$, and $90-95 \%$. The metric accounting for the percentage of video degradation $V_{d}$ can be defined as follows:

$$
\begin{gathered}
M=\{T O V, S F P\} \\
V_{d}^{M}=\frac{100 \cdot \sum_{i=0}^{n-1} k_{i}}{n}\left\{\begin{array}{lll}
k_{i}=1 & \text { if } & M_{i} \geq 1 \\
k_{i}=0 & \text { if } & M_{i}=0
\end{array}\right.
\end{gathered}
$$

where $n$ is the total number of time stamps for the test (i.e., 40 time stamps for the TOV and 120 for the SFP), $i$ is the time stamp index, and $k$ is a boolean variable assigned 1 when $M$ (either the TOV or SFP) is exceeded at least one time in the time stamp $i$ (visually perceived image distortion).

The observer is located at a recommended distance of $1.2 \mathrm{~m}$ to $1.8 \mathrm{~m}$ from the TV receiver (in Fig. 1). The total observation time is 40 minutes divided into 4 sets of 10 minutes for avoiding visual fatigue, minimizing the probability of missing the occurrence of a noticeable picture distortion. 


\section{B. LTE-A protection ratio}

In order to validate and improve the accuracy of the experimental assessment of the LTE-A QoS degradation caused by broadcasting networks, the protection ratio of the LTE-A service has to be determined. The protection ratio correspond to an LTE-A signal degradation to the limit of $10 \%$ of the Data-Block-Error-Rate (DBLER). This value is determined for a frequency offset up to $14 \mathrm{MHz}$. An offset beyond the channel bandwidth is considered for identifying any effect caused by out-of-band emissions from adjacent channels during later QoS measurments in a real scenario. The methodology for experimentally determining the protection ratio in laboratory conditions is based on the ITU recommendations BT.2215-72018 [20] and BT.1368-13-2017 [19].

\section{Experimental assessment of the LTE-A QoS degradation caused by broadcasting interference}

The goal of our experiment is to quantify the effect of the interference on the QoS of a real LTE-A network. Fig. 2 shows the experimental setup.

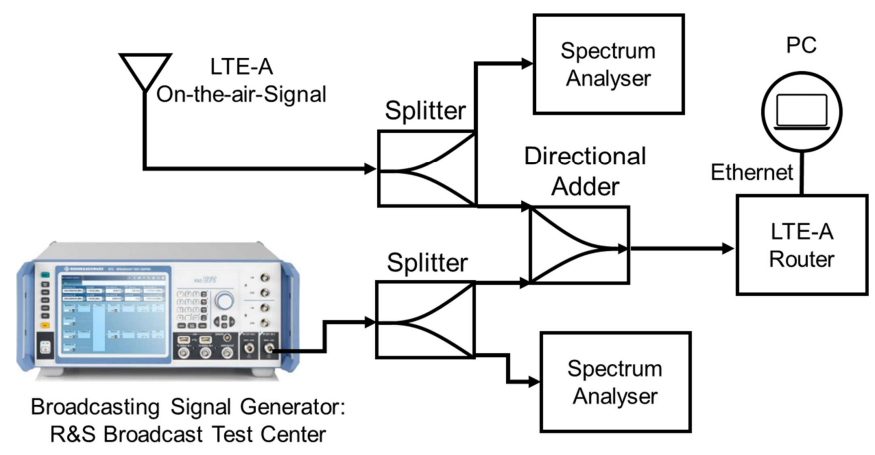

Fig. 2. Experimental setup: interference from broadcasting to a real LTE-A network.

We assume the LTE-A network is based on cognitive radio technology and is operating in the same channel of a broadcasting service. For the experimental setup, it is assumed that cognitive radio technology allows an LTE-A BS to operate in the same frequency band of a broadcasting transmitter. An LTE-A signal from an operator is inserted in a closed loop and added to a digital television broadcasting signal, either DTMB (Digital Terrestrial Multimedia Broadcast) or DVB-T2 (in Fig. 2).

For assessing the effect caused by the LTE-A BS connection management algorithm, the experiment is performed for three different operators, i.e., two LTE-A operators in Belgium (Belgian Operators 1 and 2) and one in Cuba (Cuban Operator 1). The signals are constantly monitored (Spectrum Analysers in Fig. 2). Through an LTE-A router, the signal is demodulated and a computer measures the effective data throughput at the IP (Internet Protocol) layer level. First, the signal to noise ratio is chosen to be high enough for allowing the maximum throughput the BS can provide to the user at a certain instant of time. Here, we verify that the BS load is maintained with a variation lower than $10 \%$. For the Belgian Operator 1 and
Cuban Operator 1 the load of the BS is in the range of $40-50 \%$ during the tests. For the Belgian Operator 2 the load of the BS was higher than $80 \%$. The signal (LTE-A)-to-interference (DTMB or DVB-T2) ratio is decreased in steps of $1 \mathrm{~dB}$, and the throughput is recorded at each step. The DTMB and DVBT2 signal are configured in the Broadcasting Signal Generator according to the actual transmissions in the region. The used throughput by the video streaming is higher than $90 \%$.

\section{RESULTS}

\section{A. Broadcasting QoE degradation caused by LTE-A interfer- ence}

Fig. 3 shows the percentage of video degradation for DTMB and DVB-T2 as a function of the interference caused by the traffic of the LTE-A BS in the investigated suburban areas.

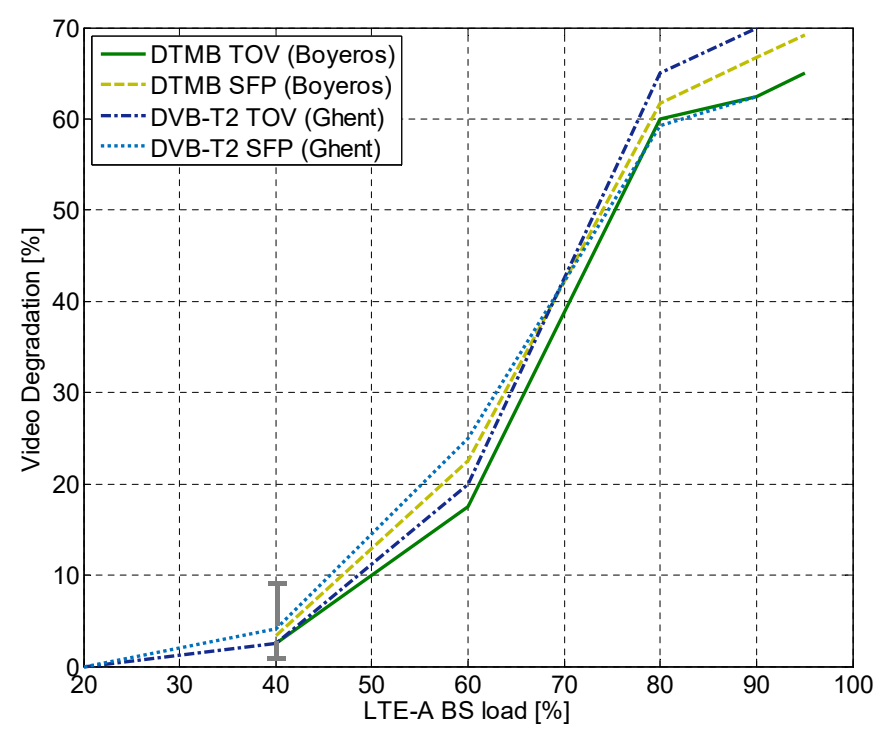

Fig. 3. Perceived video degradation by the DTMB and DVB-T2 users as a function of the traffic load of the LTE-A BS.

In the graph (Fig. 3) we denote the video degradation results for the highest limit of the peak traffic load for each load range. During the test it was constantly monitored that the BS load was in the predefined range for each experiment. In addition the environmental propagation conditions were experimentally validated and monitored in the course of the experiments. In Ghent, the peak traffic load of the BS was never higher than 90\% and in Boyeros was never lower than $40 \%$.

For the highest traffic load in the LTE-A BS, the perceived video distortion in the broadcasting service is higher than $60 \%$ of the time for both broadcasting standards and metrics, being up to $70 \%$ in the case of the TOV metric for DVB-T2 (in Fig. 3). This difference is not caused by the broadcasting technologies itself, but the differences of each scenario. Below a traffic load of $40 \%$ the video distortion leads to zero (Fig. 3). However, when the percentage of load of the LTE-A BS is lower, the standard deviation of the video degradation increased to $10 \%$, due to the fact of a higher burst of errors 
caused by more random traffic from the LTE-A users, and lower signalization traffic (fewer users connected to the BS).

The protection contour of the broadcasting transmitters in the cognitive radio standards and literature were defined assuming typical propagation conditions and generalizing the broadcasting transmitters setup. However, this protection limits for the broadcasting transmitter coexisting with cognitive radio technologies might not be enough for avoiding harmful interference to the broadcasting service in some critical conditions. In general, the broadcasting transmitters have not any protection mechanism for providing QoS data feedback to the broadcasting transmitter, the cognitive radio access controller or geolocation database [13].

\section{B. LTE-A protection ratio and frequency offset}

Fig. 4 shows the protection ratio $[\mathrm{dB}]$ for LTE-A as a function of the frequency offset.

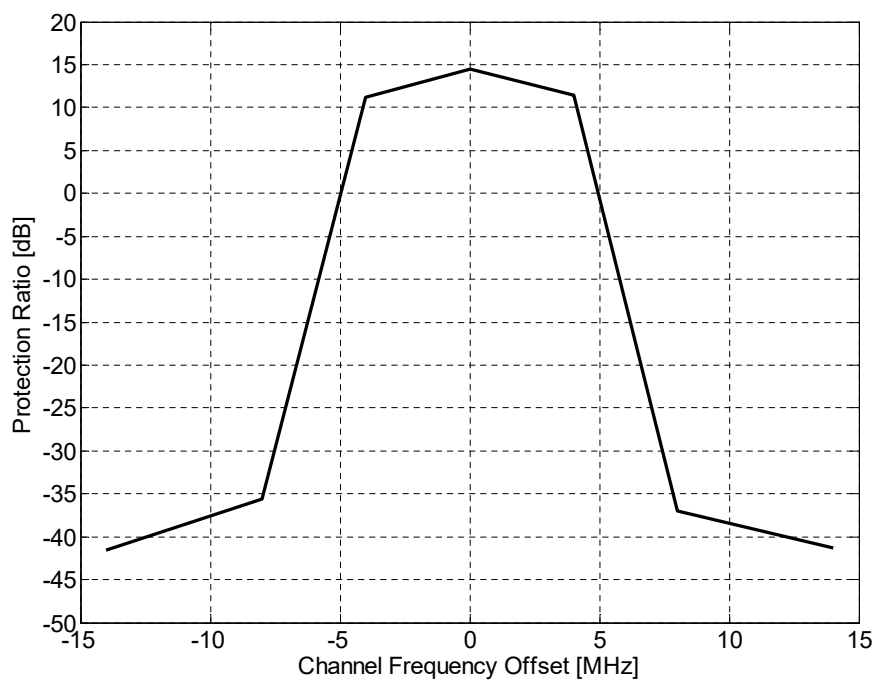

Fig. 4. Protection ratio as a function of the frequency offset for LTE-A (10 MHz channel bandwidth)

For a $0 \mathrm{MHz}$ deviation of the interfering signal the protection ratio is approximately $15 \mathrm{~dB}$. For this case a $10 \%$ DBLER is expected for the QoS experimental assessment. However, for an offset of $10 \mathrm{MHz}$, the isolation between channels has an additional protection of $35 \mathrm{~dB}$. In the real scenarios evaluated, the LTE-A emission mask provides a minimum isolation of $41 \mathrm{~dB}$ at the $\mathrm{N}+1$ and $\mathrm{N}-1$ channel. Hence, the adjacent channel interference (from other LTE-A BSs) will have no significant effect in the experimental assessment of the QoS.

\section{LTE-A QoS degradation caused by broadcasting interfer- ence}

Fig. 5 shows the degradation of the throughput of the LTEA user device interfered by a broadcasting signal in the real scenarios. The 10\% Data Block Error Rate (DBLER) corresponds to a throughput degradation of 10 to $15 \%$ compared to the maximum throughput without interference, except for

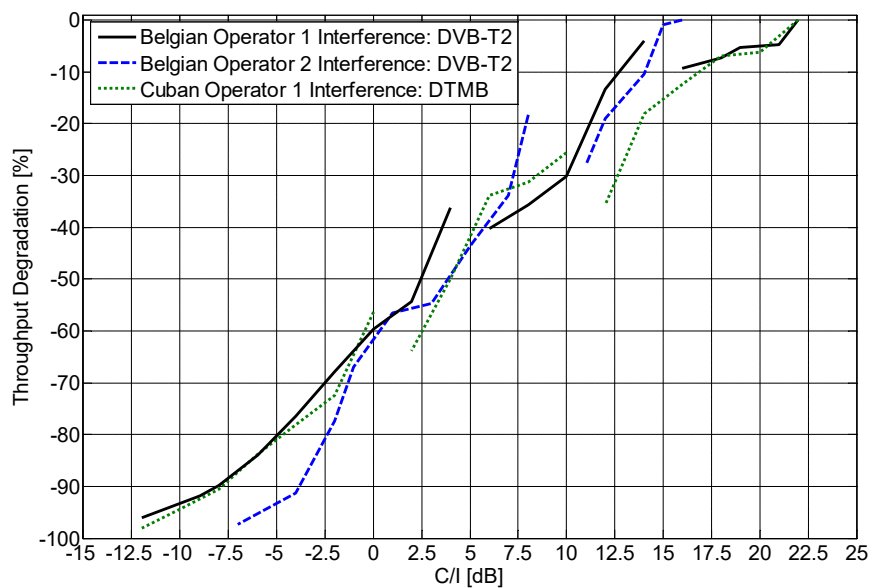

Fig. 5. Degradation of the maximum throughput of the LTE-A CPE as a function of the $\mathrm{C} / \mathrm{I}$.

the Belgian Operator 2 (in Fig. 5). The higher load of the BS of the Belgian Operator 2 leads to a higher self-interference. The highest $\mathrm{C} / \mathrm{I}$ ratio achieved in the experiment, $16 \mathrm{~dB}$, is just $1.5 \mathrm{~dB}$ from the $10 \%$ Data Block Error Rate (DBLER).

For all the operators (in Fig. 5), there is a range of 2 to $5 \mathrm{~dB}$ margin where the effective throughput is not degraded despite a lower $\mathrm{C} / \mathrm{I}$. The discontinuities in the throughput degradation as a function of the C/I are caused by the LTEA BS management of the Physical Resource Block (PRB) and the Modulation and Coding Schemes (MCS). Most of the day the LTE-A BS is not congested with a traffic load below $50 \%$. Hence, there is a wide range of resources for accommodating users to PRBs with lower interference and assigning a more robust MCS, mitigating the effect of the interference. The discontinuity range in the throughput as a function of the $\mathrm{C} / \mathrm{I}$ offers an opportunity for the dynamic interference management of a cognitive radio-based network with a centralized access controller.

\section{COnclusions}

In this paper, we investigate the coexistence of DTMB and DVB-T2 with LTE-A, assuming its cognitive radio operation, in two real suburban scenarios. If an LTE-A BS is controlled by a distributed spectrum management system, the LTE-A devices cause severe harmful interference (higher than 60\%) to the broadcasting network.

There is up to $5 \mathrm{~dB} \mathrm{C} / \mathrm{I}$ margin that does not worsen the LTE-A network QoS. A centralized access controller might take advantage of this and reduce the interference to broadcasting services without affecting the LTE-A QoS.

\section{REFERENCES}

[1] G. Hattab and M. Ibnkahla, "Multiband Spectrum Access: Great Promises for Future Cognitive Radio Networks," Proceedings of the IEEE, vol. 102, no. 3, pp. 282 - 306, 2014.

[2] A. Arteaga and A. Navarro, "Availability of TV White Spaces Using Spectrum Occupancy Information and Coverage Maps," IEEE LATIN AMERICA TRANSACTIONS, vol. 14, no. 6, pp. 2588 - 2591, 2016. 
[3] D. A. Arista-Ramirez, M. Cardenas-Juarez, U. Pineda-Rico, A. Arce and E. Stevens-Navarro, "Spectrum Occupancy Measurements in the Sub-6 GHz Band for Smart Spectrum Applications," in Latin-American Conference on Communications (LATINCOM), Guadalajara, Mexico, 2018.

[4] M. Hoyhtya, A. Mammela, M. Eskola, M. Matinmikko, J. Kalliovaara and e. al., "Spectrum Occupancy Measurements: A Survey and Use of Interference Maps," IEEE Communications Surveys and Tutorials, vol. 18, no. 4, pp. 2386 - 2414, 2016.

[5] Shared Spectrum Company, "General Survey of Radio Frequency Bands - $30 \mathrm{MHz}$ to $3 \mathrm{GHz}$," Vienna, Virginia, 2010.

[6] V. Valenta, R. Marsalek, G. Baudoin and M. Villegas, "Survey on Spectrum Utilization in Europe: Measurements, Analyses and Observations," 5th International ICST Conference on Cognitive Radio Oriented Wireless Networks and Communications, 2010.

[7] J. van de Beek, J. Riihijarvi and A. Achtzehn, "TV White Space in Europe," IEEE Transactions on Mobile Computing, vol. 11, no. 2, pp. 178-188, 2011.

[8] ETSI, “TR 103067 Reconfigurable Radio Systems (RRS); Feasibility study on Radio Frequency (RF) performance for Cognitive Radio Systems operating in UHF TV band White Spaces,” ETSI, France, 2013.

[9] F. Granelli, P. Pawelczak, R. Venkatesha Prasad, K. P. Subbalakshmi, R. Chandramouli, J. A. Hoffmeyer, and H. S. Berger. Standardization and research in cognitive and dynamic spectrum access networks: IEEE SCC41 efforts and other activities. IEEE Communications Magazine, 48(1):71-79, 2010.

[10] ETSI, Report TR 102 907: Reconfigurable Radio Systems (RRS); Use Cases for Operation in White Space Frequency Bands. ETSI, France, 2013.

[11] Q. Chen, G. Yu, H. M. Elmaghraby, J. Hamalainen, and Z. Ding. Embedding LTE-U within Wi-Fi Bands for Spectrum Efficiency Improvement. IEEE Network, 31(2):72-79, 2017.

[12] Ericsson, Ericsson and Intel to show live demo of $4 \mathrm{G}+5 \mathrm{G}$ dynamic spectrum sharing at MWC 2019. https://www.ericsson.com/en/pressreleases/2019/2/ericsson-and-intel-to-show-live-demo-of-4g-5gdynamic-spectrum-sharing-at-mwc-2019, 2019.

[13] R. Martinez Alonso, D. Plets, E. Fontes Pupo, M. Deruyck, L. Martens G. Guillen Nieto and W. Joseph, "IoT-Based Management Platform for Real-Time Spectrum and Energy Optimization of Broadcasting Networks," Wireless Communications and Mobile Computing, vol. 2018, 2019.

[14] V. Popescu, M. Nitti, D. Calaver, M. Razvan Ardelean and M. Fadda "QoS Evaluation for Indoor Broadcasting Services using an IoT platform," in BMSB, Valencia, Spain, 2018.

[15] Standarization Administration of the PRC, "GB 20600: Framing Structure, Channel Coding and Modulation for Digital Television Terrestrial Broadcasting System (DTMB)," Chinise National Standard, Beijing, China, 2006.

[16] A. Martínez Alonso, R. Martínez Alonso and G. Guillén Nieto, "Parámetros de calidad de recepción del estándar DTMB en $6 \mathrm{MHz}$," in IEEE Biennial Congress of Argentina (ARGENCON), Bariloche, Argentina, 2014.

[17] ETSI, "ETSI EN 302 755: Digital Video Broadcasting (DVB); Frame Structure channel coding and modulation for a second generation digital terrestrial television broadcasting system (DVB-T2)," EBU-ETSI, Cedex, France, 2012.

[18] ITU-R, "Report BT.2035-2: Guidelines and techniques for the evaluation of digital terrestrial television broadcasting systems including assessment of their coverage areas," ITU-R, Geneva, Switzerland , 2011.

[19] ITU-R, "Recommendation BT.1368: Planning criteria, including protection ratios, for digital terrestrial television services in the VHF/UHF bands," ITU-R, Geneva, Switzerland, 2017.

[20] ITU-R, "Report BT.2215: Measurements of protection ratios and overload thresholds for broadcast TV receivers," ITU-R, Geneva, Switzerland, 2018. 